Revista Electrónica Complutense de Investigación en Educación Musical ISSNe: 1698-7454

http://dx.doi.org/10.5209/RECIEM.58813

\title{
Las bandas y corales juveniles como recurso para el desarrollo integral de los adolescentes ${ }^{1}$.
}

\author{
Salvador Oriola ${ }^{2}$; Josep Gustems ${ }^{3}$; Gemma Filella ${ }^{4}$
}

Recibido: 7 de febrero de 2018 / Aceptado: 4 de julio de 2018

Resumen: Los conceptos adolescencia y práctica musical están estrechamente relacionados por la funcionalidad que desempeña la música en la vida de los adolescentes. El presente estudio, centrado en el fenómeno asociativo de las bandas y las corales juveniles de la Comunidad Valenciana y Cataluña, respectivamente, tiene como fin describir y analizar el perfil socioemocional de los adolescentes que integran este tipo de agrupaciones. Para ello se ha aplicado un protocolo de cuestionarios ( $a d$ hoc, LID y MOT) en una muestra de 660 adolescentes. Los resultados obtenidos confirman que en las agrupaciones musicales juveniles se genera un clima social muy favorable donde los adolescentes, además de aprender música, obtienen múltiples beneficios derivados del gozo estético, amistades, sentimiento de pertenencia, etc., todo ello contribuirá el desarrollo integral de los adolescentes.

Palabras clave: bandas juveniles; corales juveniles; adolescente; desarrollo integral; liderazgo; motivación académica; competencias socioemocionales.

\section{[en] Youth bands and choirs as a resource for the integral development of adolescents.}

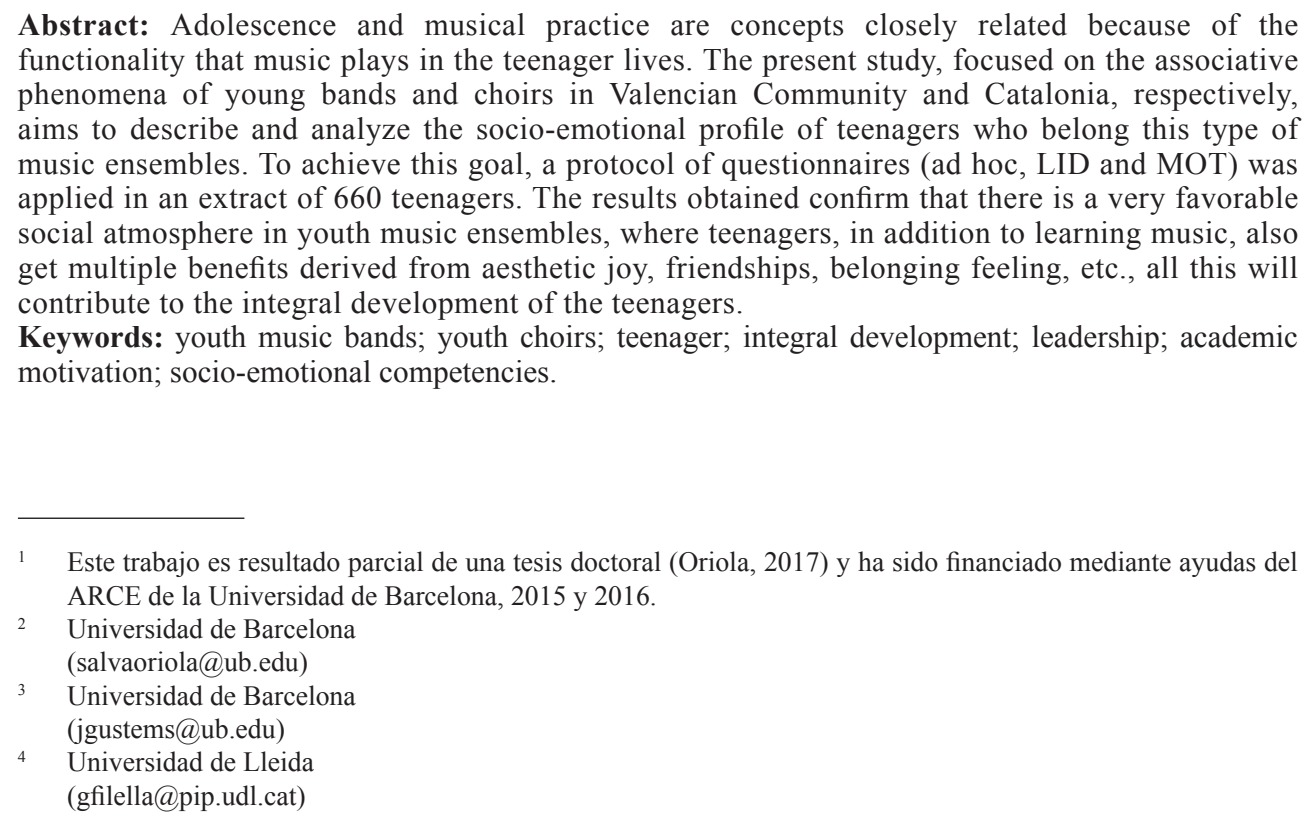

Abstract: Adolescence and musical practice are concepts closely related because of the functionality that music plays in the teenager lives. The present study, focused on the associative phenomena of young bands and choirs in Valencian Community and Catalonia, respectively, aims to describe and analyze the socio-emotional profile of teenagers who belong this type of music ensembles. To achieve this goal, a protocol of questionnaires (ad hoc, LID and MOT) was applied in an extract of 660 teenagers. The results obtained confirm that there is a very favorable social atmosphere in youth music ensembles, where teenagers, in addition to learning music, also get multiple benefits derived from aesthetic joy, friendships, belonging feeling, etc., all this will contribute to the integral development of the teenagers.

Keywords: youth music bands; youth choirs; teenager; integral development; leadership; academic motivation; socio-emotional competencies.

1 Este trabajo es resultado parcial de una tesis doctoral (Oriola, 2017) y ha sido financiado mediante ayudas del ARCE de la Universidad de Barcelona, 2015 y 2016.

2 Universidad de Barcelona

(salvaoriola@ub.edu)

3 Universidad de Barcelona

(jgustems@ub.edu)

$4 \quad$ Universidad de Lleida

(gfilella@pip.udl.cat) 
Sumario. 1. Introducción. 2. Objetivos. 3. Método. 3.1. Participantes. 3.2. Instrumentos. 3.3. Análisis de datos. 4. Resultados y discusión. 4.1. Dimensión académica-musical. 4.2. Dimensión socioemocional. 5. Conclusiones. 6. Referencias bibliográficas. 7. Anexo

Cómo citar: Oriola, S., Gustems, J. y Filella, G. (2018). Las bandas y corales juveniles como recurso para el desarrollo integral de los adolescentes, en Revista Electrónica Complutense de Investigación en Educación Musical, 15, 153-173.

\section{Introducción}

Música y adolescencia siempre han formado un binomio muy elocuente debido a las múltiples funciones que la música desempeña en la vida diaria de los adolescentes. En la actualidad los adolescentes utilizan y consumen más música que en ninguna época anterior debido, principalmente, a la inclusión masiva de los dispositivos móviles en la cultura propia de estas edades. El uso de móviles conectados a internet les permite acceder a todo tipo de música desde cualquier lugar y en cualquier momento, razón por la cual la funcionalidad de la música ha ido en aumento, tanto en cantidad como en diversidad (Williams, 2007). Sin embargo, los adolescentes de la Comunidad Valenciana y Cataluña, desde hace más de medio siglo, han tenido la oportunidad de acceder y conocer un amplio repertorio de todo tipo de música, gracias a la educación musical no formal que ofrecen las numerosas bandas y corales juveniles existentes en ambos territorios (Aviñoa, 2009; Leal, 2014).

Sendos fenómenos asociativos musicales se originaron a mediados del s. XIX, aunque las causas de su génesis fueron dispares. En Cataluña, Josep Anselm Clavé fundó las primeras corales populares con el objetivo de fomentar la sociabilidad y moralizar a la clase obrera en Barcelona, sacando a los hombres de las tabernas y cafés para culturalizarlos y ensalzar sus condiciones morales. El origen coral catalán, asociado a unas notables connotaciones sociales, políticas e ideológicas, benefició tanto a obreros como patrones pues se intentó conseguir una determinada simbiosis entre el trabajo y la fiesta, en el que la tolerancia y la integración social sirvieran como recursos para hacer frente a los problemas de clases (Carbonell, 2000b). El origen de las bandas en la Comunidad Valenciana desempeñó otras funciones como: proporcionar ocio musical y amenizar las fiestas populares (fallas, moros y cristianos, procesiones...) de ciudades y pueblos; ofrecer una educación musical destacable; democratizar el repertorio culto; etc. (Asensi, 2013).

Un siglo después, a mediados del siglo XX, empezaron a fundarse las primeras corales infantiles y juveniles en Cataluña y las primeras bandas juveniles en la Comunidad Valenciana, con el paso de los años ambos fenómenos han evolucionado y se han afianzado hasta llegar a la actualidad, en que el censo de la Federación de Sociedades Musicales de la Comunidad Valenciana (FSMCV, 2018) está integrado por más de 370 bandas juveniles y en el caso de Cataluña, a falta de un censo oficial, se estima que hay registradas en las distintas federaciones y confederaciones de corales catalanas ${ }^{5}$ más

Las federaciones y confederaciones de corales catalanas son: Corals Joves de Catalunya (CJC, 2018), Federació Catalana d'Entitats Corals (FCEC, 2018), Federació Cors Clavé (FCC, 2018), Secretariat de Corals Infantils de Catalunya (SCIC, 2018), Federació Catalana de Pueri Cantores (FCPC, 2018), Moviment Coral Català (MCC, 2018) y Agrupació Coral de les Comarques de Girona (ACCG, 2018). 
de 200 agrupaciones corales juveniles. Un reciente estudio de Fernández, Corraliza y Ferreres (2017) además de confirmar dichas cifras, las complementa con aspectos sociodemográficos, organizativos y de repertorio del fenómeno coral español. Estos datos son un claro indicativo de la holgada tradición musical existente en ambos territorios, en los que este tipo de agrupaciones, además de ofrecer una educación musical de tipo no formal, son consideradas como símbolos culturales e identitarios, es decir, forman parte de la idiosincrasia y la cultura propia de cada territorio (Carbonell, 2000a; Cohen, 1997). En la Comunidad Valenciana no se concibe un pueblo sin su respectiva banda de música y lo mismo ocurre en Cataluña con las corales, son patrimonio de cada población y a la vez de cada autonomía, donde sus habitantes se sienten orgullosos e identificados con ellas. Incluso en el senado recientemente se ha hecho eco de la importancia de esta tipología de agrupaciones, con la publicación de una declaración institucional de reconocimiento de la actividad coral como bien cultural de nuestra sociedad y de apoyo de las iniciativas que persigan su fomento e implantación, así como las investigaciones en torno a ella (BOCG, 2018).

Así pues, conocida la importante tarea socio-cultural que desempeña esta tipología de agrupaciones, es necesario e interesante indagar sobre los beneficios tanto formativos como socioemocionales que obtienen los jóvenes músicos que las integran. Pese a que bandas y corales se asemejan a priori en aspectos como la citada tarea socio-cultural, la preparación colectiva a través de ensayos o la consecución de objetivos artísticos; difieren en otras características como las funciones que desempeñaron las primeras agrupaciones existentes, la formación técnico-musical de sus integrantes, los recursos económicos y humanos necesarios para el funcionamiento de cada agrupación o la dedicación de tiempo que invierten sus componentes. Por esta razón la presente investigación se centra en conocer las diferencias y similitudes que se establecen entre los componentes de las bandas juveniles de la Comunidad Valenciana y los cantores de las corales juveniles de Cataluña, haciendo especialmente hincapié en su vertiente socioemocional.

Los adolescentes que integran ambas tipologías de agrupaciones, además de recibir una educación musical de tipo no formal adquieren toda una serie de hábitos, valores y competencias extramusicales en un clima social muy saludable (Fernández, 2014; Ferrer, 2011). En las últimas décadas, un significativo número de estudios científicos se han centrado principalmente en conocer el desarrollo de competencias académicas en aquellos jóvenes que practican música, así pues, existen numerosas investigaciones empíricas en las que se comparan los resultados académicos obtenidos por alumnos con estudios musicales con los de un grupo control, verificando que el grupo experimental obtiene mejores resultados en habilidades específicas relacionadas por ejemplo con el aprendizaje del lenguaje (Patel e Iverson, 2007; Schellenberg y Weis, 2013), la lectura (Gordon, Fehd y McCandliss, 2015; Tierney y Kraus, 2013), las matemáticas (Hallam, 2016), la creatividad (Antonietti y Colombo, 2014) o el desarrollo de competencias básicas y el rendimiento académico general (Andreu, 2012; Reyes, 2010).

Así mismo, en los últimos años se ha empezado a indagar sobre el desarrollo de competencias socioemocionales, es decir, cómo la práctica musical en grupo puede repercutir positivamente en el conocimiento y el buen uso de las emociones para una mejora del bienestar tanto personal como social (Calderón, 2014; O’Reilly y Duque, 2015; Peñalba, 2017; Rabinowitch, Cross y Burnard, 2012). La investigación empírica relacionó la participación en este tipo de agrupaciones musicales con numerosas 
capacidades de realización personal y social y variables relacionadas con las habilidades emocionales, como por ejemplo: el bienestar psicosocial, el trabajo en equipo y la cooperación, la autoestima y la motivación, el desarrollo de habilidades sociales y emocionales, o la identidad personal (Campbell, Connell, y Beegle, 2007; Colson, 2012; Hinshaw, Clift, Hulbert, y Camic, 2015; Kirrane, O’Connor, Dunne y Moriarty, 2017; Kokotsaki y Hallam, 2007; Mellor, 2013).

Siguiendo esta última línea de investigación y tomando como núcleo las bandas y corales juveniles de la Comunidad Valenciana y Cataluña, respectivamente, el presente estudio pretende conocer cómo es el perfil socioemocional de los adolescentes que integran este tipo de agrupaciones.

Los rasgos socioemocionales de cada individuo vendrán definidos por el grado de desarrollo de las competencias socioemocionales, las cuales se definen como "el conjunto de conocimientos, capacidades, habilidades y actitudes necesarias para comprender, expresar y regular de forma apropiada los fenómenos emocionales" (Bisquerra, 2003, p. 22). Según el modelo teórico-práctico del GROP (2018), este tipo de competencias se estructuran en torno a cinco grandes dimensiones: conciencia emocional (conocer e identificar las emociones propias y las de los demás), regulación emocional (manejar y controlar las emociones y las respuestas a éstas de forma efectiva), autonomía personal (características y elementos relacionados con la autogestión), competencias sociales (establecer y mantener buenas relaciones interpersonales), y competencias para la vida y el bienestar (adopción de comportamientos apropiados y responsables para hacer frente de forma eficaz a problemas cotidianos de la vida). Cada una de estas cinco dimensiones está conformada por diferentes subcompetencias, algunas de las cuales mantienen una estrecha relación con aspectos relacionados con el grado de motivación académica o la capacidad de liderazgo.

El estudio del perfil socioemocional, complementado con estos dos últimos aspectos (motivación y liderazgo) nos ayudará a tener una visión más holística de la realidad intangible que genera este fenómeno musical colectivo, así como su contribución en la formación integral de sus componentes.

\section{Objetivos}

El objetivo general que se pretende alcanzar con la realización del presente estudio es:

- Conocer, describir y comparar los perfiles socioemocionales de los integrantes que forman parte de las bandas juveniles de la Comunidad Valenciana y los coros juveniles de Cataluña.

La consecución de dicho objetivo, de carácter general, se llevará a cabo a través de los siguientes objetivos específicos:

- Examinar dimensiones académico-musicales y socioemocionales de los participantes: estudios musicales, experiencia, opiniones personales, relaciones interpersonales, nerviosismo, competencias emocionales, liderazgo, motivación, etc.

- Establecer similitudes y diferencias entre el perfil de los participantes de las dos tipologías de agrupaciones participantes: bandas y corales. 


\section{Método}

La metodología utilizada para la realización de la presente investigación consiste en una metodología no experimental ex post facto, de tipo exploratorio, descriptivo y comparativo, pues se pretende a partir de los resultados obtenidos mediante la aplicación de diferentes cuestionarios a una población determinada (adolescentes músi$\cos$ ), caracterizar el fenómeno de las agrupaciones musicales juveniles indicando sus rasgos más definitorios o característicos.

\subsection{Participantes}

La muestra de la investigación está conformada por un total de 660 músicos integrantes de agrupaciones musicales juveniles, concretamente de 10 bandas juveniles de la Comunidad Valenciana y 10 corales juveniles de Cataluña. El proceso de selección de las 20 agrupaciones participantes se realizó a partir de un exhaustivo análisis del censo de agrupaciones existentes en ambos territorios. Para ello se tuvieron en cuenta aspectos como la ubicación geográfica y la densidad de la población donde se ubica cada agrupación, el número de integrantes que las conforman, los años de antigüedad, etc. Pese a tratarse de una muestra no probabilística de tipo intencional, se ha realizado un esfuerzo deliberado por obtener una muestra "representativa", es decir, se han seleccionado agrupaciones musicales supuestamente típicas para obtener así unos resultados que reflejen o se acerquen a la realidad del fenómeno de las agrupaciones musicales estudiadas.

Así pues, de los 660 participantes, 367 (55,6\%) pertenecían a una banda y 293 $(44,4 \%)$ a una coral. De todos ellos, $237(35,9 \%)$ eran hombres y $423(64,1 \%)$ mujeres, tal y como queda reflejado en la figura 1, donde se indica la frecuencia y el porcentaje de músicos integrantes en función de las variables género y tipo de agrupación.

De acuerdo con la figura 1, en las bandas el porcentaje de hombres y mujeres están a la par, en cambio en las corales, predomina de forma significativa la presencia de mujeres. Este predominio del género femenino en las corales, tal y como afirma Elorriaga (2011), es producto de una confluencia de diversos factores, como son la muda de la voz en estas edades, la heterogeneidad de agrupaciones, razones de género, etc.

La edad media de los participantes era 14,26 años $(S D=2,29)$ y no existen diferencias estadísticamente significativas entre las dos tipologías de agrupaciones participantes. La gran mayoría de alumnos, concretamente el 66,9\% $(\mathrm{n}=441)$, se encontraba cursando la E.S.O. (Educación Secundaria Obligatoria); un 20,9\% (n = 138) Bachillerato; un 6,1\% $(\mathrm{n}=40)$ Educación Primaria; un 5,5\% $(\mathrm{n}=36)$ estudios universitarios; y el $0,6 \%(n=4)$ restante ciclos formativos.

Con la finalidad de comparar los resultados obtenidos por la muestra expuesta (músicos adolescentes) se confeccionó otro grupo formado por 655 participantes adolescentes no músicos procedentes del mismo entorno socioeconómico. No se encontraron diferencias significativas en cuanto a género y edad entre los dos grupos, lo cual garantizó la adecuada composición del grupo de no músicos para comparar los resultados y analizar los objetivos estipulados en la presente investigación. 


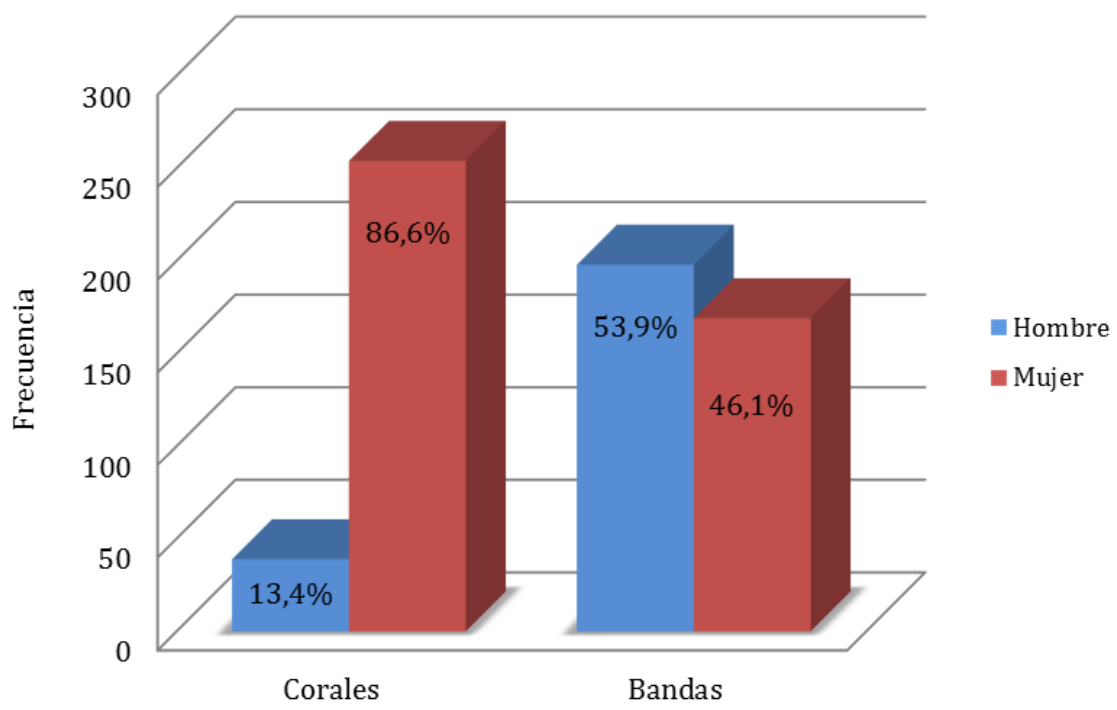

Figura 1. Músicos adolescentes en función del género y tipo de agrupación

\subsection{Instrumentos}

Con el fin de alcanzar los objetivos propuestos se ha aplicado un protocolo de cuestionarios conformado por: un cuestionario elaborado ad hoc, que recoge datos relacionados con las dimensiones académico-musicales y socioemocionales; el cuestionario LID (Ávila de Encío, 2012) para evaluar las capacidades de liderazgo; y el cuestionario MOT sobre el grado de motivación académica (Sáez, 2008).

Debido a la inexistencia de instrumentos psicométricos que se adapten a las particularidades del fenómeno estudiado, ha sido necesario diseñar el cuestionario ad hoc anteriormente referido. Para la creación de dicho cuestionario se elaboró un primer borrador, cuyo contenido fue validado a través de la técnica de juicio de expertos, basada en la evaluación, por parte de especialistas en la materia, de la expresión (relevancia, claridad, discriminación y bipolaridad) y adecuación (calidad técnica, representatividad y coherencia) de los items planteados en el cuestionario y los objetivos que se pretenden alcanzar a través de su aplicación (Hernández, Fernández y Baptista, 1998). En dicho proceso de validación participaron 10 expertos de diferentes disciplinas (profesores doctores de la Facultad de Educación de la Universidad de Barcelona, profesores doctores de la Facultad de Educación, Psicología y Trabajo Social de la Universidad de Lleida, directores de coros y bandas, profesores de secundaria y profesores de escuelas de música y conservatorios). A partir de las correcciones, objeciones y consejos ofrecidos por todos ellos se confeccionó un segundo borrador que se aplicó a modo de prueba piloto a 5 adolescentes instrumentistas y 5 adolescentes cantores, para poder así subsanar pequeños errores y verificar su funcionalidad. 
En este estudio, el coeficiente de fiabilidad Alfa de Cronbach calculada para esta escala ha sido de 0.81 ,

El cuestionario definitivo (ver Anexo) consta de un total de 19 preguntas, 5 de ellas abiertas $\left(\mathrm{n}^{\mathrm{o}} 1,5,6,8\right.$ y 16a), 9 de elección múltiple ( $\mathrm{n}^{\circ} 2,3,4,7,9,15$, 16,17 y 18), y 6 con una escala tipo Likert de 0 a 10 puntos $\left(n^{\circ} 10,11,12,13\right.$, 14 y 19). Las preguntas propuestas se han estructurado en torno a tres grandes dimensiones: personal (edad, género y estudios), académica-musical (estudios musicales, experiencia, lugar de aprendizaje, etc.) y socioemocional (relación con compañeros y director, emociones experimentadas, nerviosismo, etc.). Una vez aplicado el ???

Mediante el cuestionario LID (Ávila de Encío, 2012) se pretende evaluar la capacidad de liderazgo que poseen los participantes. Dicho cuestionario está formado por 15 frases enunciativas, las cuales se valorarán a través de una escala psicométrica tipo Likert con 5 alternativas. A partir de la suma total de la puntuación otorgada a cada ítem se obtendrá un resultado global, según el cual se establecen tres tipologías de individuos: sin capacidad de liderazgo (personas que carecen de dotes de mando); con iniciativa propia pero sin capacidad de influenciar (personas que pese a mostrar una sólida personalidad, no son capaces de movilizar e influir a los demás de modo suficiente); y personas con capacidad de liderazgo (seguras de sí mismas, que tienen una alta capacidad para tomar decisiones e influir en los demás).

El último cuestionario que conforma el protocolo aplicado es el MOT (Sáez, 2008), un cuestionario cuya finalidad es conocer la predisposición y el grado de motivación que los alumnos adolescentes tienen en torno a su formación académica. Este cuestionario consta de 25 ítems y una escala de valoración tricotómica (verdadero, falso o dudoso) a partir de la cual se obtendrá una puntuación global relativa a un único factor: el grado de motivación académica. Según la suma total de los resultados obtenidos, la motivación de los participantes podrá ser alta, moderada o baja.

\subsection{Análisis de datos}

El vaciado y el análisis de los datos recogidos en estos 3 cuestionarios se ha llevado a cabo con la ayuda de los programas Microsoft Excel 2016 y el paquete estadístico SPSS (Statistical Package for the Social Sciences, versión 24.0). Estos dos programas han permitido calcular porcentajes, medias, desviación típica, asimismo, se ha realizado un análisis de varianza (ANOVA) entre los resultados obtenidos por el grupo de músicos de banda y el grupo de músicos de corales, con el fin de comparar la variabilidad de dichos resultados y conocer si la hipótesis de que existe homogeneidad entre grupos es afirmativa o, en caso contrario, sí existen diferencias estadísticamente significativas entre grupos. También se ha aplicado un ANOVA entre adolescentes integrantes de una agrupación musical y adolescentes no músicos, para comprobar si la pertenencia a alguna agrupación musical influye positivamente en la adquisición de competencias socioemocionales.

A partir del análisis estadístico, la descripción, la comparación, el contraste, la clasificación y la interpretación de toda la información obtenida se ha pasado a la reflexión y elaboración de las conclusiones. 


\section{Resultados y discusión}

De acuerdo con la estructura del cuestionario ad hoc utilizado en la investigación, los resultados se han estructurado en torno a dos grandes dimensiones: académicamusical y socioemocional.

\subsection{Dimensión académica-musical}

Las escuelas de música, con un $68,5 \%(\mathrm{n}=452)$, son el lugar donde la mayoría de los participantes adquieren sus conocimientos musicales, seguidas en segundo lugar por los conservatorios oficiales, con un $20,6 \%(n=136)$. Un $6,5 \%(n=43)$ de los participantes empezaron sus estudios en una escuela de música y posteriormente pasaron a un conservatorio. Finalmente, encontramos un grupo reducido de 4,4\% (n =29) que afirmaban haber adquirido sus conocimientos musicales de otras formas. Tal y como afirman otras investigaciones (Díaz, 2002; Morant, 2013), esta significativa cantidad de datos se dieron y son factibles debido a la extensa red de escuelas de músicas existentes tanto en la Comunidad Valenciana como en Cataluña, pues prácticamente todas las poblaciones poseen una escuela de música local por la que pasan la mayoría de alumnos que conforman las agrupaciones musicales presentes en ambos territorios.

La media de edad en la que empezaron a estudiar música es de 6,36 años (SD = $2,54)$, sin que haya diferencias estadísticamente significativas entre bandas y corales. Un 33,3\% $(n=220)$ empezaron sus estudios musicales antes de los 6 años (Educación Infantil); un 39,4\% $(\mathrm{n}=260)$ empezaron entre los 6 y 7 años (ciclo inicial de Educación Primaria); un 17,9\% $(\mathrm{n}=118)$ entre los 8 y 9 años (ciclo medio de Educación Primaria); un 5,5\% $(\mathrm{n}=36)$ entre los 10 y 11 años (ciclo superior de Educación Primaria); y finalmente el 3,9\% $(\mathrm{n}=26)$ restante después de los 11 años (Educación Secundaria y Universidad). El inicio de los estudios musicales suele coincidir con los últimos cursos de educación infantil y el primer ciclo de educación primaria, periodo en el que algunos niños empiezan a realizar actividades extraescolares.

En cuanto a la experiencia que poseen los participantes como integrantes de una agrupación musical, de acuerdo con los resultados del ANOVA $(\mathrm{F}(3,85)=56,79 ; * \mathrm{p}$ $<0,01)$ sí que existen diferencias significativas entre ambos tipos de agrupaciones, ya que la media obtenida en las bandas es de 3,90 años $(\mathrm{SD}=0,24)$ mientras que en las corales alcanza los 5,52 años $(\mathrm{SD}=3,26)$. Tal y como se refleja en la figura 2, en las bandas el mayor porcentaje de años de experiencia se acumula durante los 5 primeros años para disminuir posteriormente de forma considerable; en cambio, en las corales se observa la convivencia de miembros con muy distintos años de experiencia.

Estos resultados son un claro reflejo del sistema estructural y organizativo que sigue cada una de las dos tipologías de agrupaciones. Las bandas juveniles tienen como objetivo principal la preparación musical de sus integrantes; éstos permanecen en ellas unos pocos años para adquirir formación y experiencia en la práctica colectiva y, posteriormente, se incorporan a las agrupaciones adultas. En cambio, la pertenencia a una coral infantil o juvenil se da desde que se inician los estudios musicales y suele ser un fin en sí mismo, es decir, los componentes no asisten con el objetivo de progresar para acceder a una agrupación superior, aunque sí que existen algunas sociedades corales con extensa y consolidada trayectoria que siguen el mismo procedimiento que las bandas (Oriola, 2017). 


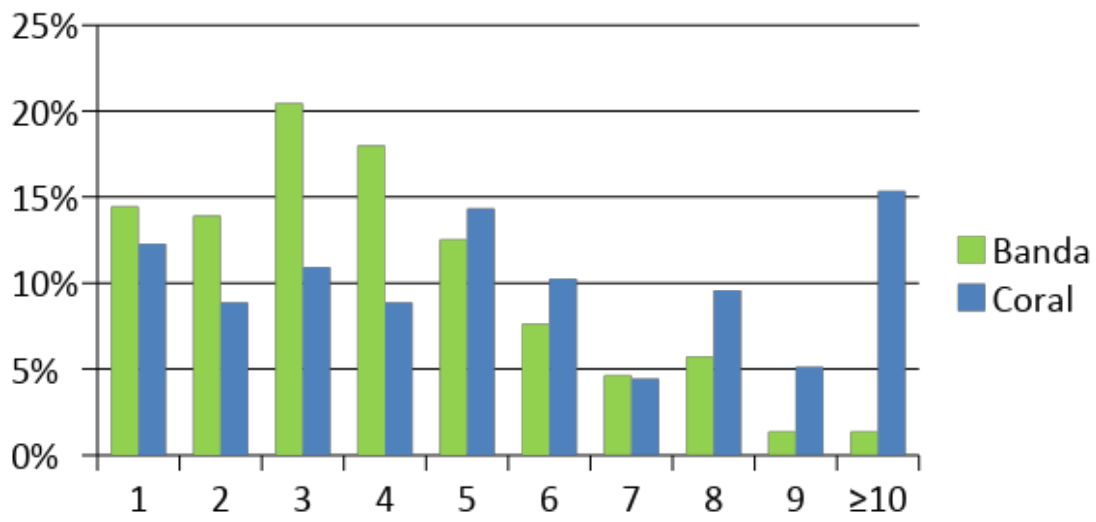

Figura 2. Años de experiencia según el tipo de agrupación musical

Con respecto a los motivos principales por los que los participantes alegan pertenecer a una agrupación musical, también se encuentran diferencias significativas entre ambas agrupaciones $(\mathrm{F}(3,85)=6,29 ; * \mathrm{p}=0,012)$. En las corales, un $91,8 \%(\mathrm{n}=269)$ afirma pertenecer a su formación por el placer que le aporta y porque asisten sus amigos, y solo el $8,2 \%(n=24)$ restante porque quiere dedicarse profesionalmente; sin embargo, en las bandas, pese a que un $74,1 \%$ $(\mathrm{n}=272)$ también indica como principales motivos el placer y las relaciones interpersonales, encontramos un destacado $25,9 \%(\mathrm{n}=95)$ que lo ve como una posible salida laboral (ver figura 3). Estos datos concuerdan globalmente con los de Kokotsaki y Hallam (2007) en lo referente a la sensación de inclusión social, sentido de pertenencia, hacer amigos y trabajo en equipo de los miembros de agrupaciones musicales juveniles.

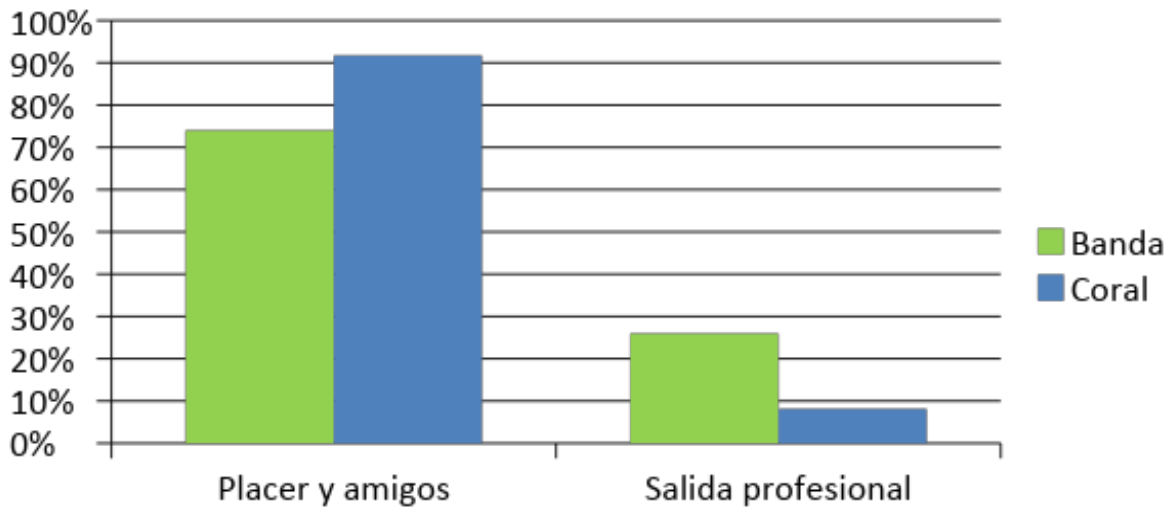

Figura 3. Motivos alegados para pertenecen a una agrupación musical 
Estrechamente relacionado con los motivos de pertenencia se encuentra aquello que más les gusta de sus respectivas agrupaciones. En las bandas, con un 49,9\% ( $\mathrm{n}=183)$, y sobre todo en las corales, con un $66,2 \%(n=194)$, el motivo preferido indicado son las relaciones sociales que se establecen en el seno de cada agrupación. De acuerdo con el ANOVA estos resultados presentan diferencias significativas entre agrupaciones $(\mathrm{F}(3,85)$ $=8,5 ; * \mathrm{p}<0,01)$. Así mismo, en las bandas, al existir un notable porcentaje de miembros que tienen como objetivo dedicarse profesionalmente, se valoran los conciertos y actos oficiales con un $37,7 \%(n=137)$ como la opción que más les gusta, frente al $20,1 \%(n=$ 59) de las corales. Los ensayos y el aprendizaje de nuevos repertorios reciben en ambos tipos de agrupaciones una valoración baja pero similar, en torno al 6\% cada ítem.

Sobre la opinión del repertorio habitual que se interpreta, la valoración que hacen los componentes de ambos tipos de agrupaciones es similar, sin que existan diferencias significativas entre grupos (la media obtenida es de 7,89; $\mathrm{SD}=1,18$ ). Éste es un claro indicativo de que en ambos tipos de agrupaciones se interpreta un repertorio variado que gusta notablemente a la gran mayoría de músicos. Estos resultados van en la línea de investigaciones realizadas en agrupaciones musicales juveniles según las cuales, el repertorio que se interpreta es uno de los factores que más repercute en la motivación de sus integrantes (Brufal, 2013; Fernández, 2014).

\subsection{Dimensión socioemocional}

Como acabamos de comprobar las relaciones interpersonales son el motivo predilecto para asistir a una agrupación musical juvenil, por esta razón no es de extrañar que la valoración media otorgada sea muy alta, concretamente de 8,92 $(\mathrm{SD}=1,13)$. También se valora de manera muy positiva, con una media de $8,30(\mathrm{SD}=1,32)$, la relación personal que mantienen los músicos con sus respectivos directores musicales, independientemente del tipo de agrupación. Todo ello repercute de forma beneficiosa en el comportamiento y la actitud de todos los componentes, tal y como confirma la media de 8,49 (SD = 1,18), obtenida a partir de la pregunta sobre estos dos ítems. Estos datos son un claro reflejo de que la cohesión social en las agrupaciones musicales es muy significativa debido al trabajo en equipo y la cooperación que exige cualquier interpretación colectiva, reafirmando las palabras de Colson: "probablemente no exista una actividad que necesite más trabajo en equipo y cooperación que la que realiza una agrupación musical" (Colson, 2012, p. 146).

En relación a la ansiedad o el nerviosismo que se experimenta en una agrupación musical, no se han encontrado diferencias destacables entre agrupaciones, la media total es de 3,19 $(\mathrm{SD}=2,52)$. Un $16,4 \%(\mathrm{n}=108)$ manifiesta no experimentar ningún tipo de nerviosismo y un $64,2 \%(n=424)$ indica sentir un nerviosismo bajo, puntuado entre los valores 1 y 5 . Aproximadamente un $12,5 \%(n=83)$ padece un nerviosismo moderado entre 6 y 7 puntos, y solo un reducido $6,8 \%(n=45)$ expresa sentir un nerviosismo significativo (cuantificada entre 8 y 10). Los momentos en que se desencadena dicha ansiedad tienen relación principalmente con la responsabilidad que exige interpretar un solo $(31,2 \%, \mathrm{n}=206)$, la participación en conciertos públicos $(18,9 \%, \mathrm{n}=125)$ o en ambos momentos, es decir, la realización de un solo en un concierto $(12,9 \%, \mathrm{n}=85)$. El resto de porcentajes, significativamente menores, se refieren a momentos cuando uno no se sabe la partitura, ensayos, relación con el director, etc.

Ante la experimentación de ansiedad se propuso una pregunta relacionada con el conocimiento y uso de estrategias para poder hacerle frente. De acuerdo con los resultados, más de la mitad de músicos que reconocen ponerse nerviosos, concre- 
tamente un $58,2 \%(n=384)$ afirman no conocer o utilizar ninguna estrategia para calmarse, frente a un $25,8 \%(n=168)$ que sí lo hacen. De este último grupo, un $71,4 \%(n=120)$ especifica emplear técnicas relacionadas con la relajación (respiración consciente, concentración...), mientras que el 28,6\% $(\mathrm{n}=48)$ restante utiliza técnicas de distracción (pensar en otras cosas, contar números...)

En relación con las emociones experimentadas durante los ensayos y conciertos, debido al amplio rango de emociones existentes y con el fin de acotar dichas valoraciones, se propuso una batería con las 8 emociones más características que conforman el círculo de Russell ${ }^{6}$ (preguntas 17 y 18 del anexo), entre las que los alumnos marcarían aquellas 3 que más experimentan durante la participación con sus respectivas agrupaciones. Los resultados no muestran diferencias estadísticas significativas entre ambos tipos de agrupaciones.

En el caso de los ensayos, encontramos que la muestra total $(n=660)$ experimenta mayoritariamente emociones de valencia positiva, aunque de arousal heterogéneo, entre éstas destacan: la alegría $(99,2 \%, \mathrm{n}=655)$, la relajación $(86,8 \%, \mathrm{n}=573)$ y la euforia $(71,8 \%, n=474)$. Con un porcentaje considerablemente menor se hallan la sorpresa $(21,7 \%, n=143)$ y el aburrimiento $(12,3 \%, n=81)$. Finalmente, con menos de un 5\% encontramos emociones de valencia negativa como la pasividad, la tristeza y la angustia con un 4,5\% $(n=30), 2,4 \%(n=16)$ y $1,2 \%(n=8)$, respectivamente. Clift y Hancox (2001) así como Hinshaw, Clift, Hulbert, y Camic (2015) ya habían encontrado resultados similares respecto al aumento de las emociones de signo positivo en miembros de corales universitarias, lo que refuerza la idea de los beneficios emocionales de este tipo de actividades.

En cambio, en los conciertos, tal y como se puede comprobar en la figura 4 , pese a que la alegría continúa siendo la emoción más experimentada con un $98,9 \%(n=653)$, la euforia, junto con la sorpresa, aumentan considerablemente sus porcentajes con un $92,4 \%(n=610)$ y un $74,1 \%(n=489)$, respectivamente. En los conciertos también se produce un aumento respecto a la angustia con un $11,8 \%(\mathrm{n}=78)$ y una disminución notable de la relajación con solo un $21,4 \%$ $(\mathrm{n}=141)$, mientras que la pasividad y el aburrimiento desaparecen (ver figura 4).

Tal y como se muestra en la figura 4 , tanto en ensayos como en conciertos predominan emociones de valencia positiva (situadas en la parte derecha del gráfico), en cambio el arousal más bajo presente en los ensayos (situado en la parte inferior) se transforma en activo durante los conciertos (situado en la parte superior). La pertenencia a una agrupación musical, además de provocar emociones propias de cualquier trabajo colectivo, también lleva implícita una exposición constante a todo tipo de música, lo cual despertará constantemente las conocidas como "emociones estéticas"7, y en definitiva, todo ello contribuirá, tal y como afirman Oriola y Gustems (2016), a la mejora de la sensibilización y el bienestar personal:

Cada pieza musical afectará de una manera concreta a cada oyente, es decir, una misma pieza provocará diferentes reacciones emocionales que dependen de fac-

6 El círculo de Russell (1980) consiste en un instrumento para la clasificación de las emociones que combina dos dimensiones principales: la valencia (valoración positiva o negativa que hace cada persona de las emociones experimentadas) y el arousal (grado de activación o excitación que experimenta cada persona cuando siente una emoción).

7 Las emociones estéticas son todas aquellas que experimenta una persona cuando percibe y valora una obra artística (musical, pictórica, teatral, escultórica, etc.). 
tores tan diversos como la edad, el estado de ánimo de cada persona, la educación recibida, el contexto, etc. Por eso, cuanta más música reciban los niños y los jóvenes, más opciones tendrán para emocionarse y mejorar su bienestar (Oriola y Gustems, 2016, p. 82).

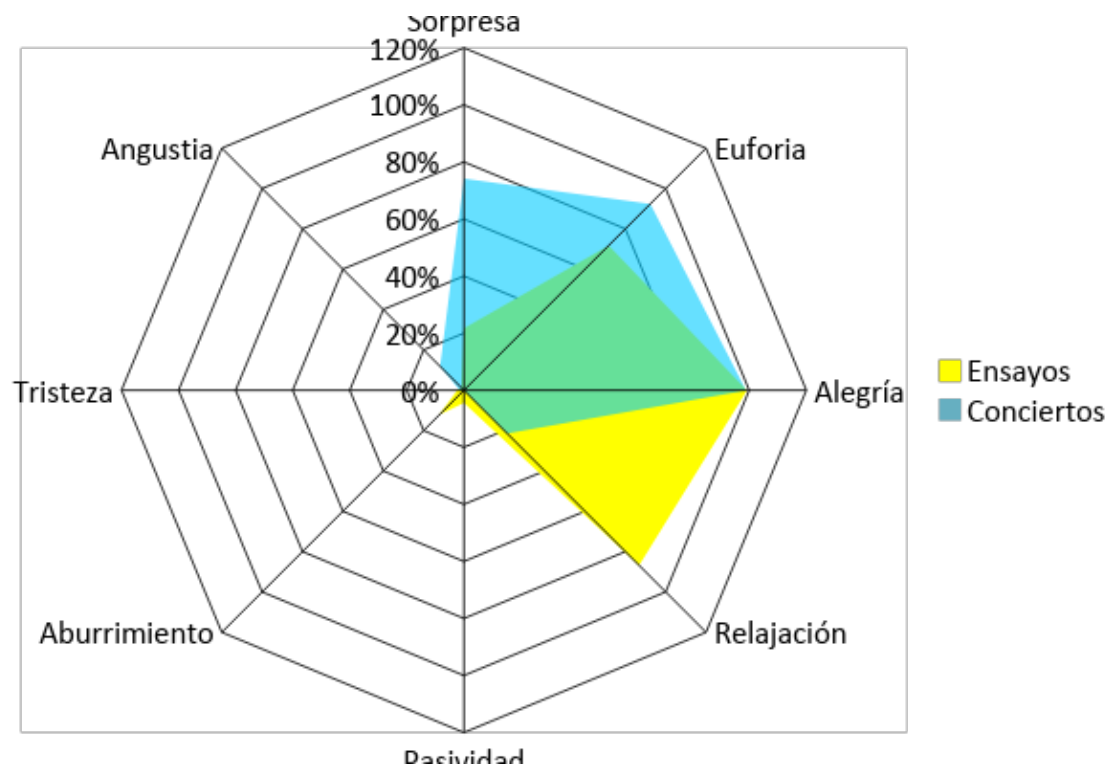

Figura 4. Comparación entre las emociones experimentadas durante los ensayos y los conciertos

Para conocer la opinión de los participantes con respecto a los beneficios socioemocionales que obtienen por participar activamente en sus respectivas agrupaciones se planteó un enunciado con 5 ítems (pregunta 19 del anexo), uno por cada una de las dimensiones emocionales propuestas por el GROP, los cuales se habían de valorar con una escala tipo Likert de 0 a 10.

No existen diferencias significativas entre ambos tipos de agrupaciones y de acuerdo con los datos obtenidos (ver tabla 1), tanto los miembros de las bandas como los de las corales opinan que participar en una agrupación musical les ayuda a adquirir y desarrollar competencias emocionales, de manera notable. Tampoco se han encontrado diferencias estadísticamente destacables por lo que respecta a la variable género. Estos resultados coinciden con algunas investigaciones (Calderón, 2014; Campayo y Cabedo, 2016; Hinshaw, Clift, Hulbert, y Camic, 2015; Kokotsaki y Hallam, 2007) según las cuales la práctica musical colectiva sería un recurso que, además de proporcionar conocimientos musicales, también contribuiría a la formación socioemocional de aquellos que la llevan a cabo.

Respecto a la capacidad de liderazgo de los participantes, los resultados obtenidos a través del cuestionario LID indican que un $55 \%(\mathrm{n}=363)$ de la muestra manifiesta sentirse muy seguro de sí mismo y tener altas capacidades para ejercer de líder; un $43,2 \%(n=285)$ expresa tener unas capacidades de liderazgo moderadas; y un reducido grupo, concretamente un $1,8 \%(\mathrm{n}=12)$, indica carecer de dotes de mando. 
Tabla 1. Valoración de cada una de las dimensiones emocionales y el promedio total

\begin{tabular}{|c|c|c|c|c|}
\hline \multirow{4}{*}{$\begin{array}{c}\text { Muestra } \\
\mathrm{n}=660\end{array}$} & $\begin{array}{c}\text { Competencia } \\
\text { Emocional }\end{array}$ & $\begin{array}{c}\text { Rango } \\
\text { Mín. } \\
\text { Máx. }\end{array}$ & Media & SD \\
\cline { 2 - 5 } & Conciencia & $2 / 10$ & 7,57 & 1,64 \\
\cline { 2 - 5 } & Regulación & $0 / 10$ & 7,95 & 1,62 \\
\cline { 2 - 5 } & Autonomía & $2 / 10$ & 7,77 & 1,39 \\
\cline { 2 - 5 } & $\begin{array}{c}\text { Comp. } \\
\text { Social }\end{array}$ & $3 / 10$ & 7,93 & 1,37 \\
\cline { 2 - 5 } & $\begin{array}{c}\text { Comp. } \\
\text { Bienestar }\end{array}$ & $2 / 10$ & 8,66 & 1,43 \\
\cline { 2 - 5 } & Total & $2,2 / 10$ & 7,98 & 1,09 \\
\hline
\end{tabular}

De acuerdo con los resultados del $\operatorname{ANOVA}(F(3,85)=15,76 ; * p<0,01)$ existen diferencias estadísticamente significativas entre bandas y corales en cuanto a la capacidad de liderazgo se refiere (ver figura 6).

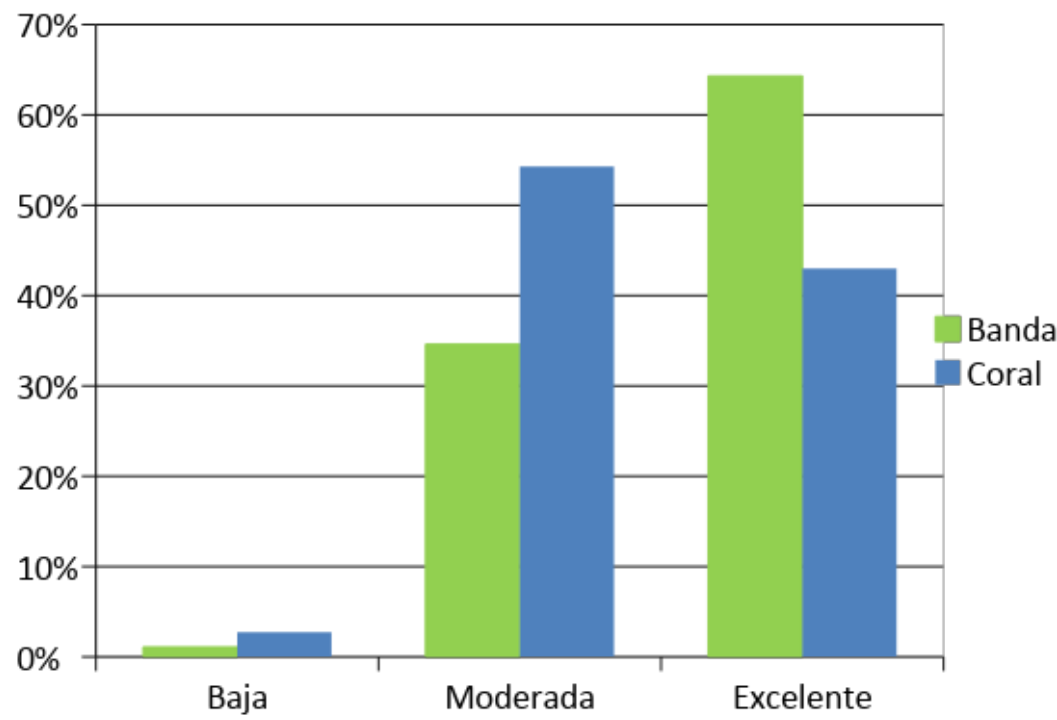

Figura 6. Resultados del cuestionario LID según el tipo de agrupación.

Tal y como se puede comprobar en la figura 6, en las bandas juveniles predominan los adolescentes con altas capacidades y habilidades para el liderazgo $(64,3 \%, n=236)$, en cambio, en las corales, el $54 \%(n=159)$ de sus integrantes indica tener unas competencias de liderazgo moderadas. No existen diferencias estadísticamente relevantes por lo que respecta a la variable de género, tanto hombres como mujeres, indistintamente del tipo de agrupación poseen unas capacidades para el liderazgo semejantes. 
La comparación entre los resultados de la muestra conformada por adolescentes músicos y el grupo de no músicos (ver tabla 2), indica que también existen diferencias estadísticamente significativas entre ambos grupos.

Tabla 2. Análisis de varianza (ANOVA) entre los resultados del LID

\begin{tabular}{|c|ccccc|}
\hline $\begin{array}{c}\text { Origen de las } \\
\text { variaciones }\end{array}$ & $\begin{array}{c}\text { Suma de } \\
\text { cuadrados }\end{array}$ & $\begin{array}{c}\text { Grados de } \\
\text { libertad }\end{array}$ & $\begin{array}{c}\text { Promedio de los } \\
\text { cuadrados }\end{array}$ & F & Sig. \\
\hline Entre grupos & 10036,22 & 1 & 10036,22 & 93,07 & $<, 001 *$ \\
\hline $\begin{array}{c}\text { Dentro de los } \\
\text { grupos }\end{array}$ & 141573,23 & 1313 & 107,82 & & \\
\hline Total & 151609,46 & 1314 & & \\
\hline
\end{tabular}

Estos resultados van en la misma línea que otros estudios como los de Calderón, Gustems y Calderón (2015) o Hallam y Creech (2010), los cuales concluyen que las competencias y habilidades relacionadas con el liderazgo se suelen adquirir y desarrollar de forma notable entre los jóvenes que estudian y practican música.

Finalmente, los resultados del cuestionario MOT para conocer el grado de motivación académica de los participantes, ponen en relevancia la alta motivación que tienen los integrantes de ambos tipos de agrupaciones. El 82,7\% (n $=546)$ de la muestra demuestra tener una alta motivación, frente a un $16,1 \%$ $(\mathrm{n}=106)$ que tiene una motivación normal y un $1,2 \%(\mathrm{n}=8)$ con una motivación muy baja. El grado de motivación académica en ambas tipologías de agrupaciones es prácticamente idéntico y de acuerdo con el ANOVA no existen diferencias estadísticamente significativas entre grupos ni tampoco entre la variable género. En cambio, en el grupo de adolescentes no músicos la motivación académica es inferior, predominando una motivación moderada tal y como se puede comprobar en la figura 7. Los resultados del ANOVA $(\mathrm{F}(3,85)=247,70 ; * \mathrm{p}<0,01)$, corroboran que existen diferencias estadísticamente notables entre los adolescentes músicos y los no músicos.

Tanto instrumentistas como cantores presentan una alta motivación académica, lo cual puede influir de forma positiva en el rendimiento y los resultados académicos, tal y como señalan algunos estudios (Andreu, 2012; Reyes, 2011). Otros estudios exponen que el alto rendimiento académico parece ser un predictor para ingresar y permanecer en una banda (Kinney, 2010). A pesar de ello, cabe remarcar que la motivación académica es un complejo proceso en el que interactúan tanto aspectos personales (intereses, capacidades...) como contextuales (amigos, familia, sociedad, cultura...) (Hallam y Creech, 2010), de ahí que se necesitaría una investigación multivariable para conocer con mayor exactitud la razón por la que existe esta diferencia estadísticamente significativa entre músicos y no músicos. 


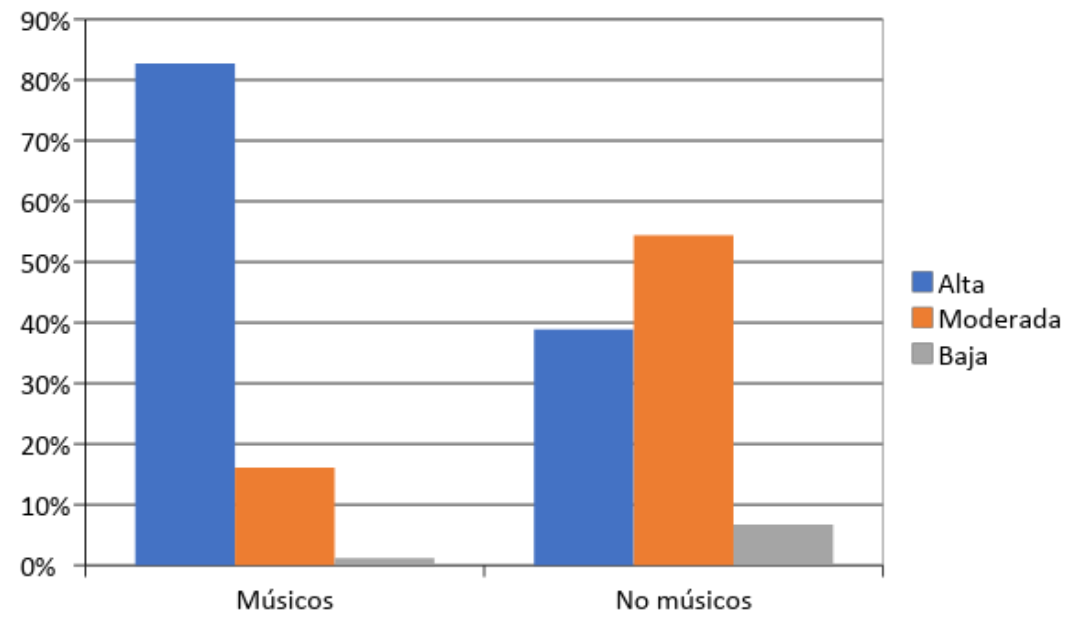

Figura 7. Resultados del cuestionario MOT

\section{Conclusiones}

En base a los resultados ofrecidos y coincidiendo con otros estudios como los citados por Carrillo, Viladot y Pérez-Moreno (2017), se puede concluir que el adolescente que forma parte de una agrupación musical adquiere y desarrolla toda una serie de capacidades y competencias que contribuirán en su formación tanto musical como personal.

Las agrupaciones musicales juveniles, indistintamente de su tipología, ofrecen un clima social valorado de forma muy positiva por sus integrantes. Éstos, además de aprender música, obtienen múltiples beneficios como gozo estético, amistades, sentimiento de pertenencia e integración, enriquecimiento personal, etc. Todo ello puede contribuir al desarrollo no solo de competencias académico-musicales sino también socioemocionales, sin grandes diferencias atribuibles a la distinta orientación histórica de ambos tipos de agrupaciones (Oriola, 2017). Así pues, no es de extrañar que los integrantes de ambos tipos de agrupaciones muestren un buen perfil competencial, con puntuaciones elevadas en relación al desarrollo de competencias emocionales, liderazgo o motivación académica.

A pesar de la similitud del perfil obtenido entre bandas y corales, existen algunas diferencias estadísticamente significativas entre algunos de los rasgos valorados como la capacidad de liderazgo o los motivos alegados para ingresar en una agrupación musical. Estas diferencias posiblemente se den debido a los distintos objetivos académico-musicales que persigue cada tipología de agrupación, y estrechamente relacionado con ello, los requerimientos musicales necesarios (lenguaje musical y técnica instrumental) para formar parte de una banda o una coral.

Las bandas juveniles en su totalidad, pese a proponer diferentes objetivos específicos que se adaptan a las particularidades de cada formación, comparten un objetivo principal que consiste en formar y motivar a sus integrantes a través de la interpretación musical colectiva y el trabajo cooperativo. Esta motivación será imprescindible 
para que los adolescentes continúen con la práctica instrumental y adquieran cierta experiencia que les permita poder incorporarse posteriormente a una banda de adultos, con un nivel musical cuanto más alto mejor. La combinación de todos estos elementos puede repercutir en el devenir de sus componentes, ya que el trabajo cualificado junto con la proposición de objetivos motivadores, además de ofrecer una buena formación musical y una posible salida laboral, también contribuirá significativamente, ya sea de forma consciente o inconsciente, al desarrollo personal de cada músico.

En el caso de las corales, la formación musical de sus integrantes generalmente no requiere de un aprendizaje musical tan específico como el exigido en las bandas; esta es la razón por la que el desarrollo de competencias profesionales en los cantores suele ser menor. Además, debido a la heterogeneidad de corales existentes, los objetivos musicales propuestos en cada agrupación son muy diversos, predominando muchas veces la dimensión social sobre la musical. Todo ello repercutirá en la adquisición de competencias tanto musicales como socioemocionales; pese a darse un desarrollo competencial significativo en sus componentes, éste suele ser inferior con respecto a los de las bandas.

Los resultados obtenidos en este estudio pueden ser relevantes para diferentes campos de conocimiento como son la educación musical, la educación emocional, la psicología de la música, la práctica instrumental colectiva, etc., aunque hay que remarcar que existen unas limitaciones que se deben tener en cuenta. La escasa literatura referente al estudio de la repercusión socioemocional que tiene la participación activa en agrupaciones musicales junto con la inexistencia de estudios sobre los fenómenos asociativos musicales existente en la Comunidad Valenciana y Cataluña, imposibilita la contrastación de los resultados obtenidos, en consecuencia, las conclusiones expuestas se han de tomar con cautela y provisionalidad, hasta que no proliferen estudios similares con los que se puedan cotejar. En segundo lugar, la homogeneidad estructural y logística que caracteriza a las bandas juveniles de la Comunidad Valenciana sí que puede servir para conocer con detalle cómo son estas agrupaciones y el perfil de sus integrantes, es decir, se pueden considerar los resultados obtenidos como representativos. En cambio, la pluralidad de corales existentes y la heterogeneidad que se da en cuanto a su organización y los objetivos que persiguen, quizás requiera de un estudio más amplio y pormenorizado; los resultados obtenidos serían como una foto panorámica sobre el perfil de sus integrantes, aunque sería interesante poder hacer un zoom y profundizar en la interacción de variables como el género, la procedencia socioeconómica..., a través de una investigación con una muestra más extensa.

Aun así, esperamos que los resultados obtenidos puedan contribuir a la mejora en la toma de decisiones relacionadas con la organización y el trabajo llevado a cabo en el seno de este tipo de agrupaciones por agentes tan diversos como directores musicales, juntas directivas, representantes, etc., y que también sirvan como estímulo para la realización de futuros estudios, que se encarguen de indagar y demostrar la importancia de este tipo de formaciones, tanto a nivel académico-musical como a nivel socioemocional.

\section{Referencias bibliográficas}

Agrupació Coral de les Comarques de Girona (ACCG). (2018). Portal de la Agrupació Coral de les Comarques de Girona. Recuperado de https://federaciocoraldegirona.wordpress. com/ 
Andreu, M. (2012). L'assoliment de les competències bàsiques en alumnes de centres integrats de primària i música. Tesis doctoral. Universitat Autònoma de Barcelona. Recuperado de http://hdl.handle.net/10803/96516

Antonietti, A. y Colombo, B. (2014). Musical thinking as a kind of creative thinking. En E. Shiu (Ed.), Creativity Research. An inter-disciplinary and multi-disciplinary research handbook (pp. 233-246). Nueva York: Routledge.

Asensi, E. (2013). Música, mestre!! Les bandes valencianes en el tombant del segle XIX. Valencia: Universitat de València.

Ávila de Encío, C. (2012). Cuestionario para valorar la capacidad de liderazgo (LID). Recuperado de https://bit.ly/2ubpi45

Aviñoa, X. (2009). El cant coral als segles XIX i XX. Catalan Historical Review, 2, 203-212.

Bisquerra, R. (2003). Educación emocional y competencias básicas para la vida. Revista de Investigación Educativa (RIE), 21 (1), 7-43.

BOCG. (2018). Boletín Oficial de las Cortes Generales, 209, 36-37.

Brufal, J. D. (2013). Estudio cuantitativo de la trayectoria en educación musical de los músicos de banda. Eufonía, 57, 65-75.

Calderón, D. (2014). La práctica en grupo como camino hacia el bienestar de los adolescentes. En J. Gustems (Ed.), Arte y bienestar (pp. 75-83). Barcelona: Universitat de Barcelona.

Calderón, D., Gustems, J. y Calderón, C. (2015). Las competencias personales y sociales en las colonias musicales. Revista Electrónica Complutense de Investigación en Educación Musical, 12, 53-72. DOI: http://dx.doi.org/10.5209/rev_RECIEM.2015. v12.47157

Campbell, P., Connell, C. y Beegle, A. (2007). Adolescents' expressed meanings of music in and out of school. Journal of Research in Music Education, 55 (3), 220-236.

Campayo, E. A. y Cabedo, A. (2016). Música y competencias emocionales: posibles implicaciones para la mejora de la educación musical. Revista Electrónica Complutense de Investigación en Educación Musical, 13, 124-139. DOI: http://dx.doi.org/10.5209/ RECIEM.51864

Carbonell, J. (2000a). El cant coral. En X. Aviñoa (Dir.), Història de la Música Catalana, Valenciana i Balear. Del Romanticisme al Nacionalisme, Vol. 3 (pp. 147-186). Barcelona: Edicions 62.

Carbonell, J. (2000b). Josep Anselm Clavé i el naixement del cant coral a Catalunya (18501874). Cabrera de Mar: Galerada.

Carrillo, C., Viladot, L. y Pérez-Moreno, J. (2017). Impacto de la educación musical: una revisión de la literatura científica. Revista Electrónica Complutense de Investigación en Educación Musical, 14, 61-74. DOI: http://dx.doi.org/10.5209/RECIEM.54828

Clift, S. M. y Hancox, G. (2001). The Perceived Benefits of Singing Findings from Preliminary Surveys of a University College Choral Society. The Journal of the Royal Society for the Promotion of Health, 121 (4), 248-256. DOI: 10.1177/146642400112100409

Cohen, R. S. (1997). The Musical Society Community Bands of Valencia, Spain: A global study of their administration, instrumentation, repertoire and performance activities. Tesis doctoral inédita. Chicago: Northwestern University.

Colson, J. F. (2012). Conducting and rehearsing the instrumental music ensemble: scenarios, priorities, strategies, essentials, and repertoire. Maryland: Scarecrow Press.

Corals Joves de Catalunya (CJC). (2018). Portal de les Corals Joves de Catalunya. Recuperado de http://www.coralsjoves.cat/ 
Díaz, M. (2002). La música en la educación primaria y en las escuelas de música: la necesaria coordinación. Tesis doctoral. Universidad del País Vasco-Euskal Herriko Unibertsitatea. Recuperado de http://edtb.euskomedia.org/5833/

Elorriaga, A. J. (2011). La continuidad del canto durante el periodo de la muda de la voz. Tesis doctoral. Madrid: Universidad Autónoma de Madrid. Recuperado de http://hdl. handle.net/10486/7818

Federació Catalana d'Entitats Corals (FCEC). (2018). Portal de la Federació Catalana d'Entitats Corals. Recuperado de http://www.fcec.cat/

Federació Catalana de Pueri Cantores. (2018). Portal de la Federació Catalana de Pueri Cantores. Recuperado de http://www.puericantores-cat.com/

Federació de Cors de Clavé (FCC). (2018). Portal de la Federació de Cors de Clavé. Recuperado de http://www.josepanselmclave.cat/

Federació de Societats Musicals de la Comunitat Valenciana (FSMCV). (2018). Portal de la Federació Societats Musicals de la Comunitat Valenciana. Recuperado de http://www. fsmcv.org/

Fernández, N. S. (2014). Las agrupaciones corales y su contribución al bienestar de las personas. Percepción de las aportaciones del canto coral. Tesis doctoral. Madrid: Universidad Carlos III. Recuperado de http://e-archivo.uc3m.es/handle/ 10016/18185

Fernández, N., Corraliza, J. A. y Ferreras, S. (2017). Las agrupaciones corales en España: espacios para la convivencia y la educación musical. Revista Internacional de Educación Musical, 5, 17-29. DOI: 10.12967/RIEM-2017-5-p017-029

Ferrer, R. (2011). El cant coral infantil i juvenil educa en valors, hàbits i competències. Tesis doctoral. Universitat de Girona. Recuperado de http://hdl.handle.net/ 10803/77826

Gordon, R. L., Fehd, H. M. y McCandliss, B. D. (2015). Does Music Training Enhance Literacy Skills? A Meta-Analysis. Frontiers in Psychology, 6, 1-16. DOI: http://doi. org/10.3389/fpsyg.2015.01777

GROP. (2018). Grup de Recerca en Orientació Psicopedagògica. Recuperado de http:// www.ub.edu/grop/

Hallam, S. (2016). The impact of actively making music on the intellectual, social and personal development of children and young people: a summary. Voices: A World Forum for Music Therapy, 16 (2). Recuperado de https://normt.uib. no/index.php/voices/article/ view/884/725

Hallam, S. y Creech, A. (Eds.). (2010). Music Education in the 21st Century in the United Kingdom. Londres: Institute of Education, University of London.

Hernández, R., Fernández, C. y Baptista, P. (1998). Metodología de la Investigación. México: McGraw Hill.

Hinshaw, T., Clift, S., Hulbert, S. y Camic, P. M. (2015). Group singing and young people's psychological well-being. International Journal of Mental Health Promotion, 17 (1), 4663. DOI: $10.1080 / 14623730.2014 .999454$.

Kinney, D. W. (2010). Selected nonmusic predictors of urban students' decisions to enroll and persist in middle school band programs. Journal of Research in Music Education, 57 (4), 334-350. DOI: 10.1177/0022429409350086

Kirrane, M., O’Connor, C., Dunne, A. M. y Moriarty, P. (2017). Intragroup processes and teamwork within a successful chamber choir. Music Education Research, 19 (4), $357-$ 370. http://dx.doi.org/10.1080/14613808.2016.1166195

Kokotsaki, D. y Hallam, S. (2007). Higher education music students' perceptions of the benefits of participative music making. Music Education Research, 9 (1), 93-109. DOI: $10.1080 / 14613800601127577$ 
Leal, J. de D. (Ed.). (2014). Las Bandas de Música de la Comunitat Valenciana. Valencia: Editorial Gules.

Mellor, L. (2013). An Investigation of Singing, Health and Well-being as a Group Process. British Journal of Music Education, 30 (2), 177-205. https://doi.org/10.1017/ S0265051712000563

Morant, R. (2013). Planteamientos de educación formal en enseñanzas no regladas de música: las Escuelas de Música de las Sociedades Musicales Valencianas. Revista Electrónica LEEME, 31, 79-106. Recuperado de: http://musica.rediris.es/leeme

Moviment Coral Català (MCC). (2018). Portal del Moviment Coral Català. Recuperado de http://www.mcc.cat/

O’Reilly, M. y Duque, E. J. (2015). Las agrupaciones corales como estrategia de formación de competencias para trabajo en equipo en las organizaciones: una perspectiva comparativa. Suma de Negocios, 6 (13), 92-97.

Oriola, S. (2017). Las agrupaciones musicales juveniles y su contribución al desarrollo de competencias socioemocionales. El fenómeno de las bandas en la Comunidad Valenciana y los coros en Cataluña. Tesis doctoral. Lleida: Universitat de Lleida. Recuperado de http://hdl.handle.net/10803/405944

Oriola, S. y Gustems, J. (2016). El procés emocional d'escoltar i produir música. Temps d'Educació, 50, 69-85.

Patel, A. D. e Iverson, J. R. (2007). The linguistic benefits of musical abilities. Trends in Cognitive Sciences, 11, 369-372.

Peñalba, A. (2017). La defensa de la educación musical desde las neurociencias. Revista Electrónica Complutense de Investigación en Educación Musical, 14, 109-127. DOI: http://dx.doi.org/10.5209/RECIEM.54814

Rabinowitch, T., Cross, I. y Burnard, P. (2012). Long-term musical group interaction has a positive influence on empathy in children. Psychology of Music, 41 (4), 484-498.

Reyes, M. C. (2010). El rendimiento académico de los alumnos de primaria que cursan estudios artísticomusicales en la comunidad valenciana. Tesis doctoral. Valencia: Universitat de Valencia. Recuperado de http://hdl.handle.net/10803/81333

Russell, J. A. (1980). A circumplex model of affect. Journal of Personality and Social Psychology, 39, 1161-1178.

Sáez, A. (2008). Cuestionario de motivación académica para los alumnos. Recuperado de http://perso.wanadoo.es/angel.saez/pagina nueva_95.htm

Schellenberg, E. G. y Weiss, M. W. (2013). Music and cognitive abilities. En D. Deutsch (Ed.), The psychology of music (pp. 499-550). Amsterdam: Elsevier.

Secretariat de Corals Infantils de Catalunya (SCIC). (2018). Portal del Secretariat de Corals Infantils de Catalunya. Recuperado de http://www.scic.cat/

Tierney, A. y Kraus, N. (2013). Music training for the development of reading skills. En M. M. Merzenich, M. Nahum y T. M. Van Vleet (Eds.), Progress in Brain Research (pp. 209241). Burlington: Academic Press.

Williams, A. (2007). Portable Music and Its Functions. Nueva York: Peter Lang Publishing. 


\section{Anexo}

Cuestionario sobre dimensiones académicas y emocionales relacionadas con la música

1. Edad:

2. Sexo:

Hombre Mujer

3. Estudios generales que estás cursando en la actualidad:
E.S.O
Bachillerato
Ciclo formativo
Universidad
Ed. Primaria

4. Estudios musicales realizados en:

$\square$

Escuela de música

$\square$ Conservatorio

Autodidacta

$\square$ Otros:

5. ¿Cuantos años llevas estudiando música?

6. ¿Qué instrumento o instrumentos sabes tocar?

Principal: Secundario:

7. ¿Por qué formas parte de una coral/banda?
$\square$ Por obligación

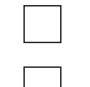
Porque te gustaría dedicarte profesionalmente a la música
Porque te gusta
Porque están tus amigos Otros:

8. ¿Cuantos años hace, en total, que eres miembro de una coral/banda?

9. ¿Qué es lo que más te gusta de tu coral/banda?
La relación con los compañeros
Los ensayos Los conciertos y actos oficiales
$\square$ Conocer nuevo repertorio
Otros:

10. ¿Cómo te motiva el repertorio que se interpreta en tu coral/banda? Valora marcando con una cruz la casilla del 0 (nada) al 10 (mucho).

\begin{tabular}{|l|l|l|l|l|l|l|l|l|l|l|}
\hline 0 & 1 & 2 & 3 & 4 & 5 & 6 & 7 & 8 & 9 & 10 \\
\hline
\end{tabular}

11. ¿Cómo es la relación personal que tienes con los compañeros/as de tu coral/banda? Valora marcando con una cruz la casilla del 0 (nada) al 10 (mucho).

\begin{tabular}{|l|l|l|l|l|l|l|l|l|l|l|}
\hline 0 & 1 & 2 & 3 & 4 & 5 & 6 & 7 & 8 & 9 & 10 \\
\hline
\end{tabular}

12. ¿Cómo es la relación personal que tienes con el director/a de tu coral/banda? Valora marcando con una cruz la casilla del 0 (nada) al 10 (mucho).

\begin{tabular}{|l|l|l|l|l|l|l|l|l|l|l|}
\hline 0 & 1 & 2 & 3 & 4 & 5 & 6 & 7 & 8 & 9 & 10 \\
\hline
\end{tabular}


13. ¿Cómo es tu actitud y tu comportamiento cuando estás en tu coral/banda? Valora marcando con una cruz la casilla del $\mathbf{0}$ (nada) al 10 (mucho).

\begin{tabular}{|l|l|l|l|l|l|l|l|l|l|l|}
\hline 0 & 1 & 2 & 3 & 4 & 5 & 6 & 7 & 8 & 9 & 10 \\
\hline
\end{tabular}

14. ¿Te pones nervioso cuando participas en tu coral/banda? Valora marcando con una cruz la casilla del 0 (nada) al 10 (mucho).

\begin{tabular}{|l|l|l|l|l|l|l|l|l|l|l|}
\hline 0 & 1 & 2 & 3 & 4 & 5 & 6 & 7 & 8 & 9 & 10 \\
\hline
\end{tabular}

15. Si es el caso, ¿en qué momentos te pones nervioso?

$\square$ Ensayos $\square$ Conciertos $\square$ Cuando tienes algún solo $\square$ Cuando no te sabes tu papel Cuando te relacionas con tus compañeros $\square$ Cuando te relacionas con tu director

16. Cuando te pones nervioso ¿utilizas alguna estrategia para calmar los nervios?

Sí -¿Cuál o cuáles?

No

17. De las siguientes emociones, marca aquellas 3 que más experimentas en los ensayos:

Alegría $\square$ Sorpresa $\square$ Tristeza $\quad \square$ Pasividad

Euforia $\square$ Agonía $\square$ Aburrimiento $\quad \square$ Relajación

18. De las siguientes emociones, marca aquellas 3 que más experimentas en los conciertos:

\begin{tabular}{|c|c|c|c|}
\hline Alegría & Sorpresa & Tristeza & Pasividad \\
\hline Euforia & Agonía & Aburrimiento & Relajación \\
\hline
\end{tabular}

19. Valora del 0 (nada) al 10 (mucho) los beneficios socioemocionales que consideras que te aporta el hecho de pertenecer a una coral/banda:

\begin{tabular}{|c|c|c|c|c|c|c|c|c|c|c|c|c|}
\hline $\mathbf{1}$ & $\begin{array}{c}\text { Me ayuda a conocer mejor } \\
\text { mis emociones y la de mis } \\
\text { compañeros }\end{array}$ & 0 & 1 & 2 & 3 & 4 & 5 & 6 & 7 & 8 & 9 & 10 \\
\hline $\mathbf{2}$ & $\begin{array}{c}\text { Me ayuda a expresar y regular } \\
\text { las emociones }\end{array}$ & 0 & 1 & 2 & 3 & 4 & 5 & 6 & 7 & 8 & 9 & 10 \\
\hline $\mathbf{3}$ & $\begin{array}{c}\text { Me motiva y me ayuda } \\
\text { a mejorar mi autoestima }\end{array}$ & 0 & 1 & 2 & 3 & 4 & 5 & 6 & 7 & 8 & 9 & 10 \\
\hline $\mathbf{4}$ & $\begin{array}{c}\text { Me ayuda a relacionarme } \\
\text { con los otros }\end{array}$ & 0 & 1 & 2 & 3 & 4 & 5 & 6 & 7 & 8 & 9 & 10 \\
\hline $\mathbf{5}$ & \begin{tabular}{c} 
Me ayuda a mejorar mi bienestar \\
\hline
\end{tabular} & 0 & 1 & 2 & 3 & 4 & 5 & 6 & 7 & 8 & 9 & 10 \\
\hline
\end{tabular}

